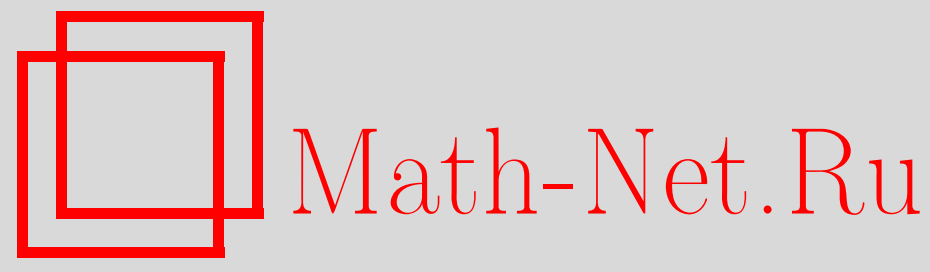

Л. Я. Савельев, С. В. Балакин, Совместное распределение числа единиц и числа 1-серий в двоичных марковских последовательностях, Дискрет. матем., 2004, том 16, выпуск $3,43-62$

DOI: https://doi.org/10.4213/dm162

Использование Общероссийского математического портала Math-Net.Ru подразумевает, что вы прочитали и согласны с пользовательским соглашением http: //www . mathnet.ru/rus/agreement

Параметры загрузки:

IP: 18.234 .156 .22

26 апреля 2023 г., 18:00:08

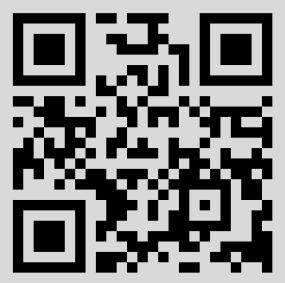




\title{
Совместное распределение числа единиц и числа 1-серий в двоичңых марковских последовательностях
}

\author{
() 2004 г. Л. Я. Савельев, С. В. Балакин
}

\begin{abstract}
Описываются распределения числа единиц, числа серий из единиц в двоичной марковской последовательности и их совместное распределение. Находятся производящие функции рассматриваемых распределений. Вычисляются средние, дисперсии и ковариации. Для них даются точные и асимптотические формулы с оценками. Выводятся формулы для нормальных приближений.

Рассматриваются соответствующие операторные уравнения. В связи с ними описывается специальная модель случайных блужданий.

Работа выполнена при поддержке Российского фонда фундаментальных исследований, проект 02-01-0946.
\end{abstract}

\section{Введение}

Первая марковская последовательность, которую описал А. А. Марков [1], была двоичной. Двоичным марковским последовательностям посвящена обширная литература. Двоичные марковские последовательности выделяются в качестве важного частного случая в большинстве работ, в которых рассматриваются марковские последовательности с конечным множествам значений [2]-[8].

Особый интерес представляют совместные распределения различных характеристик серий в двоичной марковской последовательности и первые моменты этих распределений. В статье описываются распределения числа единиц, числа серий из единиц, их первые моменты и совместное распределение. Находятся производящие функщии этих распределений. Для средних значений, дисперсий и ковариаций даются точные и приближенные формулы с оценками. Выводятся формулы для нормальных приближений.

При фиксированных первом и последнем значении рассматриваемой двоичной марковской последовательности совместные распределения числа единиц и числа серий из единиц представляются произведением некоторых биномиальных распределений. Это существенно упрощает изучение распределений и описание нормальных приближений.

Естественный переход к модели случайных блужданий связан с решением операторных уравнений специального типа и условиями их корректной разрешимости. 


\section{1. Обозначения}

1.1. Рассмотрим двоичную марковскую последовательность $\xi$ случайных переменных $\xi(k), k \geqslant 0$, с множеством значений $C=\{1,0\}$, начальным вектором $A$ и переходной матрицей $Q$ :

$$
\begin{aligned}
& A=(a, 1-a), \\
& Q=\left(\begin{array}{ll}
q_{11} & q_{10} \\
q_{01} & q_{00}
\end{array}\right)=\left(\begin{array}{cc}
p & 1-p \\
1-q & q
\end{array}\right),
\end{aligned}
$$

где

$$
\begin{aligned}
a & =\mathbf{P}\{\xi(0)=1\}, & 1-a & =\mathbf{P}\{\xi(0)=0\}, \\
q_{\alpha \beta} & =\mathbf{P}\{\xi(k+1)=\beta \mid \xi(k)=\alpha\}, & k & \geqslant 0 .
\end{aligned}
$$

Будем предполагать, что $0<p, q<1$.

1.2. Введем дополнительные обозначения

$$
d=p+q-1=\operatorname{det} Q, \quad b=\frac{1-q}{1-d}, \quad c=\frac{a-b}{1-d} .
$$

Так как предполагается, что $0<p, q<1$, то $-1<d<1$.

Равенство $d=0$ означает, что $q=1-p$, то есть вероятности переходов $0 \rightarrow 0$ и $1 \rightarrow 0$ одинаковы. При $a=p, d=0,0 \leqslant k \leqslant n-1$ получается последовательность Бернулли с параметрами $(n, p)$. Определитель $d=\operatorname{det} Q$ можно считать мерой зависимости соседних членов двоичной марковской последовательности с переходной матрицей $Q$.

Равенство $c=0$ означает, что $a=b$ и последовательность $\xi$ стационарна, то есть $\mathbf{P}\{\xi(k)=1\}=a$ при любом $k$.

Равенство $p=q$ эквивалентно тому, что $b=1 / 2$. В этом случае переходы $0 \rightarrow 0$ и $1 \rightarrow 1$ имеют одну и ту же вероятность $p$, а переходы $0 \rightarrow 1$ и $1 \rightarrow 0$ - одну и ту же вероятность $1-p$. Матрица $Q$ становится эрмитовой. Естественно назвать такую последовательность $\xi$ симметричной.

1.3. Случайные величины, равные числу единиц и числу 1-серий последовательности $\xi$ на отрезке $[0, n]$, выражаются соответственно равенствами

$$
\begin{aligned}
& x(n)=\xi(0)+\sum_{k=1}^{n} \xi(k) \\
& y(n)=\xi(0)+\sum_{k=1}^{n}(1-\xi(k-1)) \xi(k) .
\end{aligned}
$$

Сумма в выражении для $y(n)$ равна числу переходов $0 \rightarrow 1$ в последовательности $\xi$ на отрезке $[0, n]$, описывающих начало 1-серии. Часто вместо 1-серий рассматривают эти переходы. Последовательности $x(n), n \geqslant 0$, и $y(n), n \geqslant 0$, в общем случае немарковские.

Заметим также, что

$$
y(n)=x(n)-\sum_{k=1}^{n} \xi(k-1) \xi(k) .
$$

Сумма в этом выражении описывает число переходов $1 \rightarrow 1$ в последовательности $\xi$ на отрезке $[0, n]$. 


\section{2. Распределение $\xi(k)$}

2.1. По индукции легко доказать, что верно следующее выражение для $k$-й степени $Q^{k}$ матрицы $Q$ :

$$
Q^{k}=\left(\begin{array}{cc}
b+(1-b) d^{k} & 1-b-(1-b) d^{k} \\
b-b d^{k} & 1-b+b d^{k}
\end{array}\right)
$$

Распределение $P_{k}=\left(p_{k}, q_{k}\right)$ в момент $k\left(d^{k}=1\right.$ при $\left.k=0\right)$ имеет вид

$$
P_{k}=\left(b+(a-b) d^{k}, 1-b-(a-b) d^{k}\right),
$$

где

$$
\begin{aligned}
& p_{k}=\mathbf{P}\{\xi(k)=1\}=b+(a-b) d^{k} \\
& q_{k}=\mathbf{P}\{\xi(k)=0\}=1-b-(a-b) d^{k}
\end{aligned}
$$

Это распределение было вычислено еще А. А. Марковым [1].

2.2. Если, как предполагается, $|d|<1$, то предельное распределение выражается равенствами

$$
\begin{aligned}
& p_{\infty}=\lim _{k \rightarrow \infty} \mathbf{P}\{\xi(k)=1\}=b, \\
& q_{\infty}=\lim _{k \rightarrow \infty} \mathbf{P}\{\xi(k)=0\}=1-b .
\end{aligned}
$$

Таким образом, отношение $b$ вероятности перехода $0 \rightarrow 1$ к суммарной вероятности изменения значения является и предельной вероятностью значения 1 . Пусть

$$
Q^{\infty}=\left(\begin{array}{ll}
b & 1-b \\
b & 1-b
\end{array}\right), \quad R=\left(\begin{array}{cc}
1-b & -(1-b) \\
-b & b
\end{array}\right)
$$

Тогда матрица перехода через $k$ шагов выражается равенством

$$
Q^{k}=Q^{\infty}+R d^{k}
$$

Матрица $Q^{\infty}$ является бернуллиевой частью формулы. При достаточно большом $k$ подпоследовательность $\xi\left(j_{i}\right), i \geqslant 0$, с номерами $j_{i+1}-j_{i} \geqslant k$ можно приближенно считать бернуллиевой с параметром $b$.

2.3. Нетрудно вычислить ковариацию и коэффициент корреляции для случайных переменных $\xi(j), \xi(k), j \leqslant k$. Ясно, что

$$
\begin{gathered}
\mathbf{E}(\xi(j) \xi(k))=\mathbf{P}\{\xi(j)=\xi(k)=1\}=\left(b+(a-b) d^{j}\right)\left(b+(1-b) d^{k-j}\right), \\
\mathbf{D}(\xi(j))=p_{j} q_{j}=b(1-b)+(a-b)\left(1-2 b-(a-b) d^{j}\right) d^{j} .
\end{gathered}
$$

Отсюда получаются простые выражения для ковариации $\operatorname{Cov}(\xi(j), \xi(k))$ и коэффициента корреляции $K(\xi(j), \xi(k))$ :

$$
\begin{aligned}
\operatorname{Cov}(\xi(j), \xi(k)) & =p_{j} q_{j} d^{k-j}=V(\xi(j)) d^{k-j} \\
K(\xi(j), \xi(k)) & =\sqrt{\left(p_{j} q_{j}\right) /\left(p_{k} p_{k}\right)} d^{k-j}=\sqrt{\mathbf{D}(\xi(j)) / \mathbf{D}(\xi(k))} d^{k-j} .
\end{aligned}
$$


Так как $\mathbf{D}(\xi(j)) \leqslant 1$ и $\mathbf{D}(\xi(k)) \rightarrow b(1-b)$, то $K(\xi(j), \xi(k)) \rightarrow 0$ при $k-j \rightarrow \infty$, если $|d|<1$. Зависимость между переменными $\xi(j)$ и $\xi(k)$ с достаточно далекими друг от друга номерами $j, k$ мала. Это подтверждает вывод о приближенной бернуллиевости подпоследовательности в п. 2.2 .

Замечание 1. Заменяя $a=\mathbf{P}\{\xi(0)=1\}$ на $p_{k}=\mathbf{P}\{\xi(k)=1\}$, можно получить из выписанных формул для распределений, средних и дисперсий характеристик серий в последовательности $\xi(0), \ldots, \xi(n)$ аналогичные формулы для последовательности $\xi(k), \ldots, \xi(k+n)$. Так как $p_{k} \rightarrow b$ при $k \rightarrow \infty$, когда $|d|<1$, в этом случае, заменяя $a$ на $b$, можно получить приближенные формулы при больших значениях $k$.

\section{3. Совместное распределение}

3.1. Каждая из реализаций последовательности $\xi$, для которой $\xi(0)=\alpha, \xi(n)=\beta$, $x(n)=i, y(n)=j$, имеет одну и ту же вероятность

$$
p_{\alpha \beta}(i, j, n)=a^{\alpha}(1-a)^{1-\alpha} p^{i-j}(1-p)^{j-\beta}(1-q)^{j-\alpha} q^{n-i-j+\alpha+\beta} .
$$

В самом деле, из определений следует, что

$$
\begin{aligned}
p_{\alpha \beta}(i, j, n)=a^{\xi(0)}(1-a)^{1-\xi(0)} p^{\sum_{k=1}^{n} \xi(k-1) \xi(k)}(1-p)^{\sum_{k=1}^{n} \xi(k-1)(1-\xi(k))} & \\
& \times(1-q)^{\sum_{k=1}^{n}(1-\xi(k-1)) \xi(k)} q^{\sum_{k=1}^{n}(1-\xi(k-1))(1-\xi(k))} .
\end{aligned}
$$

Как легко проверить,

$$
\begin{aligned}
x(n)-y(n) & =\sum_{k=1}^{n} \xi(k-1) \xi(k), \\
y(n)-\xi(n) & =\sum_{k=1}^{n} \xi(k-1)(1-\xi(k)), \\
y(n)-\xi(0) & =\sum_{k=1}^{n}(1-\xi(k-1)) \xi(k), \\
n-x(n)-y(n)+\xi(0)+\xi(n) & =\sum_{k=1}^{n}(1-\xi(k-1))(1-\xi(k)) .
\end{aligned}
$$

Поэтому

$$
\begin{aligned}
p_{\alpha \beta}(i, j, n)=a^{\xi(0)}(1-a)^{1-\xi(0)} p^{x(n)-y(n)}(1- & p)^{y(n)-\xi(n)} \\
& \times(1-q)^{y(n)-\xi(0)} q^{n-x(n)-y(n)+\xi(0)+\xi(n)},
\end{aligned}
$$

и при $\xi(0)=\alpha, \xi(n)=\beta, x(n)=i, y(n)=j$ получается равенство (1).

Заметим, что

$$
\begin{array}{ll}
p_{\alpha \beta}(i, j, n)=0, & \text { если } i>n+1 \text { или } i<j \text { или } j>[n / 2]+1, \\
p_{\alpha \beta}(i, 0, n)=0, & \text { если } i>0
\end{array}
$$


([ $x]$ обозначает целую часть вещественного числа $x$ ). Выделяются вероятности для единственных полных 0-серии и 1-серии:

$$
\begin{aligned}
p_{\alpha \beta}(0,0, n) & =(1-\alpha)(1-\beta)(1-a) q^{n}, \\
p_{\alpha \beta}(n+1,1, n) & =\alpha \beta a p^{n} .
\end{aligned}
$$

\section{2. Рассмотрим событие}

$$
\Omega_{\alpha \beta}(i, j, n)=\{\xi: \xi(0)=\alpha, \xi(n)=\beta, x(n)=i, y(n)=j\} .
$$

Из сказанного в п. 3.1 следует, что для вероятности $P_{\alpha \beta}(i, j, n)=\mathbf{P}\left\{\Omega_{\alpha \beta}(i, j, n)\right\}$ верно равенство

$$
P_{\alpha \beta}(i, j, n)=c_{\alpha \beta}(i, j, n) p_{\alpha \beta}(i, j, n),
$$

где

$$
c_{\alpha \beta}(i, j, n)=\operatorname{card}\left(\Omega_{\alpha \beta}(i, j, n)\right) .
$$

Этот коэффициент легко вычислить, используя известную формулу для числа $s(m, k)$ разложений натурального числа $m$ на $k$ слагаемых. Для целых $k, m$ положим

$$
\left(\begin{array}{l}
m \\
k
\end{array}\right)= \begin{cases}m ! /(k !(m-k) !), & 0 \leqslant k \leqslant m, \\
0 & \text { в остальных случаях. }\end{cases}
$$

Легко видеть, что

$$
s(m, k)=\left(\begin{array}{c}
m-1 \\
k-1
\end{array}\right) .
$$

При $1 \leqslant i \leqslant n, 1 \leqslant j \leqslant[n / 2]+1$ верны равенства

$$
c_{\alpha \beta}(i, j, n)=s(i, j) s(n-i+1, j+1-\alpha-\beta)=\left(\begin{array}{c}
i-1 \\
j-1
\end{array}\right)\left(\begin{array}{c}
n-i \\
j-\alpha-\beta
\end{array}\right)
$$

( $i$ единиц распределяются по $j 1$-сериям, и $n-i+1$ нулей распределяются по $j+1-\alpha-\beta$ 0-сериям). Из (1)-(3) следует, что

$$
P_{\alpha \beta}(i, j, n)=\left(\begin{array}{c}
i-1 \\
j-1
\end{array}\right)\left(\begin{array}{c}
n-i \\
j-\alpha-\beta
\end{array}\right) a^{\alpha}(1-a)^{1-\alpha} p^{i-j}(1-p)^{j-\beta}(1-q)^{j-\alpha} q^{n-i-j+\alpha+\beta}
$$

Введем обозначения

$$
\begin{aligned}
b(k, m, x) & =\left(\begin{array}{l}
m \\
k
\end{array}\right) x^{k}(1-x)^{m-k}, \\
\chi_{\alpha \beta} & =(\alpha a+(1-\alpha)(1-a))(\beta(1-q)+(1-\beta)(1-p)) .
\end{aligned}
$$

При $1 \leqslant i \leqslant n, 1 \leqslant j \leqslant[n / 2]+1$ равенство (4) эквивалентно равенству

$$
P_{\alpha \beta}(i, j, n)=\chi_{\alpha \beta} b(j-1, i-1,1-p) b(j-\alpha-\beta, n-i, 1-q) .
$$

Замечание 2. Рассматриваемое распределение составляется из биномиальных распределений, параметрами которых служат вероятности переходов. Комбинаторные рассуждения, с помощью которых оно получено, объясняют это. Используя равенство (5) и известные асимптотические формулы для биномиального распределения, можно получать асимптотические формулы для вероятностей $P_{\alpha \beta}(i, j, n)$. 
3.3. Рассмотрим событие

$$
\Omega(i, j, n)=\{\xi: x(n)=i, y(n)=j\}=\bigcup_{\alpha, \beta} \Omega_{\alpha \beta}(i, j, n) .
$$

Из сказанного в 3.2 следует, что при $1 \leqslant i \leqslant n, 1 \leqslant j \leqslant[n / 2]+1$ для вероятности $P(i, j, n)=\mathbf{P}\{\Omega(i, j, n)\}$ верны равенства

$$
P(i, j, n)=\sum_{\alpha, \beta} P_{\alpha \beta}(i, j, n)=b(j-1, i-1,1-p) \sum_{\alpha, \beta} \chi_{\alpha \beta} b(j-\alpha-\beta, n-i, 1-q)
$$

Выделяются вероятности для единственных полных 0-серии и 1-серии

$$
P(0,0, n)=(1-a) q^{n}, \quad P(n+1,1, n)=a p^{n} .
$$

3.4. Для последовательности Бернулли $(a=p=1-q)$ верны равенства

$$
\begin{aligned}
P_{\alpha \beta}(i, j, n) & =\left(\begin{array}{c}
i-1 \\
j-1
\end{array}\right)\left(\begin{array}{c}
n-i \\
j-\alpha-\beta
\end{array}\right)(1-p)^{n-i+1} \\
P(i, j, n) & =\left(\begin{array}{c}
i-1 \\
j-1
\end{array}\right)\left(\begin{array}{c}
n-i+2 \\
j
\end{array}\right) p^{i}(1-p)^{n-i+1}
\end{aligned}
$$

\section{4. Производящие функции}

4.1. Используя формулу полной вероятности и марковское свойство, получаем систему уравнений для вероятностей $P_{\alpha \beta}(i, j, n)$ :

$$
\begin{aligned}
& P_{\alpha 1}(i, j, n)=P_{\alpha 1}(i-1, j, n-1) p+P_{\alpha 0}(i-1, j-1, n-1)(1-q), \\
& P_{\alpha 0}(i, j, n)=P_{\alpha 1}(i, j, n-1)(1-p)+P_{\alpha 0}(i, j, n-1)
\end{aligned}
$$

где $n \geqslant 1,1 \leqslant i \leqslant n+1,1 \leqslant j \leqslant[n / 2]+1$. Кроме того (граничные условия):

$$
\begin{aligned}
& P_{\alpha \beta}(i, j, 0)= \begin{cases}\alpha \beta a, & i=j=1, \\
(1-\alpha)(1-\beta)(1-a), & i=j=0, \\
0 & \text { в остальных случаях, }\end{cases} \\
& P_{\alpha \beta}(i, 0, n)=0, \quad i \geqslant 1, \\
& P_{\alpha \beta}(0, j, n)=0, \quad j \geqslant 1, \\
& P_{\alpha \beta}(0,0, n)=(1-\alpha)(1-\beta)(1-a) q^{n}, \quad n \geqslant 0, \\
& P_{\alpha \beta}(n+1,1, n)=\alpha \beta a p^{n}, \quad n \geqslant 0, \\
& P_{\alpha \beta}(i, j, n)=0, \quad i>n+1 \vee i<j \vee j>[n / 2]+1 .
\end{aligned}
$$

4.2. Рассмотрим производящие функции $h_{\alpha \beta}$ для вероятностей $P_{\alpha \beta}(i, j, n)$ :

$$
h_{\alpha \beta}(s, t, v)=\sum_{n=0}^{\infty} \sum_{i=0}^{\infty} \sum_{j=0}^{\infty} P_{\alpha \beta}(i, j, n) s^{i} t^{j} v^{n}, \quad|s|,|t| \leqslant 1, \quad|v|<1 .
$$


Используя систему (8) и граничные условия, по аналогии с [17] получаем систему уравнений

$$
\begin{aligned}
(1-p s v) h_{\alpha 1}-(1-q) s t v h_{\alpha 0} & =\alpha a s t \\
-(1-p) v h_{\alpha 1}+(1-q v) h_{\alpha 0} & =(1-\alpha)(1-a)
\end{aligned}
$$

Решая систему (10), находим, что

$$
\begin{aligned}
& h_{11}(s, t, v)=\frac{a s t(1-q v)}{1-(q+p s) v+(p q-(1-p)(1-q) t) s v^{2}} \\
& h_{10}(s, t, v)=\frac{a(1-p) s t v}{1-(q+p s) v+(p q-(1-p)(1-q) t) s v^{2}} \\
& h_{01}(s, t, v)=\frac{(1-a)(1-q) s t v}{1-(q+p s) v+(p q-(1-p)(1-q) t) s v^{2}} \\
& h_{00}(s, t, v)=\frac{1-a-(1-a) p s v}{1-(q+p s) v+(p q-(1-p)(1-q) t) s v^{2}}
\end{aligned}
$$

4.3. Из формул (11) получается формула для производящей функции $h$ вероятностей $P(i, j, n)$. Так как

$$
P(i, j, n)=\sum_{\alpha, \beta} P_{\alpha \beta}(i, j, n),
$$

суммирование (11) дает

$$
h(s, t, v)=\frac{1-a+a s t-((1-a) p+(a d-(1-a)(1-q)) t) s v}{1-(q+p s) v+(p q-(1-p)(1-q) t) s v^{2}} .
$$

Замечание 3. Формулы для производящих функщий распределений случайных переменных $x(n), y(n)$ и их совместного распределения можно получить как частные случаи общей формулы для производящих функщй совместного распределения числа единиц, числа 1-серий и максимумов длин 1-серий и 0-серий, которая была выведена в [5]. Там указаны некоторые формулы и для самого такого совместного распределения.

\section{5. Распределение числа единиц}

5.1. Пусть

$$
P(i, \cdot, n)=\sum_{j=0}^{i \wedge([n / 2]+1)} P(i, j, n) .
$$

Из (6) следует, что при $1 \leqslant i \leqslant n$ верно равенство

$$
P(i, \cdot, n)=\sum_{j=1}^{i \wedge([n / 2]+1)}\left(b(j-1, i-1,1-p) \sum_{\alpha, \beta} \chi_{\alpha \beta} b(j-\alpha-\beta, n-i, 1-q)\right) .
$$

5.2. Производящая функция $f$ для распределения $P(i, \cdot, n)$ выражается равенствами

$$
f(s, v)=h(s, 1, v)=\frac{1-a+a s-d s v}{1-(q+p s) v+d s v^{2}}
$$


5.3. Для последовательности Бернулли $(a=p=1-q)$ из (13) получается известное равенство (см. [9])

$$
P(i, \cdot, n)=\left(\begin{array}{c}
n+1 \\
i
\end{array}\right) p^{i}(1-p)^{n-i+1}
$$

Аналогичный результат дает разложение в ряд по $v$ и по $s$ производящей функции

$$
f(s, v)=\frac{1-p+p s}{1-(1-p+p s) v}
$$

\section{6. Распределение числа 1 -серий}

6.1. Пусть

$$
P(\cdot, j, n)=\sum_{i=0}^{n+1} P(i, j, n)
$$

Из (6) следует, что при $2 \leqslant j \leqslant[n / 2]+1$ верно равенство

$$
P(\cdot, j, n)=\sum_{i=1}^{n}\left(b(j-1, i-1,1-p) \sum_{\alpha, \beta} \chi_{\alpha \beta} b(j-\alpha-\beta, n-i, 1-q)\right)
$$

При $j=1$ добавляется полная 1-серия (7) и равенство имеет вид

$$
\begin{aligned}
& P(\cdot, 1, n)=a p^{n}+(a(1-p)+(1-a)(1-q)) p^{n-1} \\
& +\sum_{i=1}^{n-1}((n-i)(1-a)(1-p)(1-q)+(1-a)(1-q) q+a(1-p) q) p^{i-1} q^{n-i-1}
\end{aligned}
$$

6.2. Производящая функция $g$ для распределения $P(\cdot, j, n)$ выражается равенствами

$$
g(t, v)=h(1, t, v)=\frac{1-a+a t-(1-a) p v+(1-a p-q) t v}{1-(p+q) v+p q v^{2}-(1-p)(1-q) t v^{2}}
$$

6.3. Для последовательности Бернулли $(a=p=1-q)$ из (15) и (16) при $1 \leqslant j \leqslant$ $[n / 2]+1$ получается равенство

$$
P(\cdot, j, n)=\sum_{i=1}^{n+1}\left(\begin{array}{c}
i-1 \\
j-1
\end{array}\right)\left(\begin{array}{c}
n-i+2 \\
j
\end{array}\right) p^{i}(1-p)^{n-i+1}
$$

Производящая функщия $g$ имеет вид

$$
g(t, v)=\frac{1-p(1-t)-p(1-p)(1-t) v}{1-v+p(1-p)(1-t) v^{2}}
$$




\section{7. Среднее число единиц}

7.1. Среднее значение $\mathrm{E}(x(n))$ случайной величины $x(n)$ выражается равенствами

$$
\mathbf{E}(x(n))=\sum_{k=0}^{n} \mathbf{E}(\xi(k))=\sum_{k=0}^{n} p_{k}=\sum_{k=0}^{n}\left(b+(a-b) d^{k}\right) .
$$

Используя формулу для суммы членов геометрической прогрессии, находим, что

$$
\mathbf{E}(x(n))=(n+1) b+c\left(1-d^{n+1}\right) .
$$

Слагаемое $(n+1) b$ является биномиальной частью формулы (18). Если $a=p$ и $d=0$, то $c=0, b=1-q=p$, марковская последовательность становится бернуллиевой и $\mathbf{E}(x(n))=(n+1) p$. Слагаемое $c\left(1-d^{n+1}\right)$ является адयитивной поправкой на марковскую зависимость случайных переменных $\xi(k)$. То же равенство для средних можно получить, дифференцируя по $s$ производящую функцию $f(s, v)$, заданную равенством (14), и разлагая производную в точке $t=1$ в ряд по $v$.

7.2. Так как по предположению $|d|<1$, при $n \rightarrow \infty$

$$
\mathbf{E}(x(n)) \sim(n+1) b+c \sim n b .
$$

Это соответствует представлению о рассматриваемой двоичной марковской последовательности $\xi(1), \ldots, \xi(n)$ как об асимптотически бернуллиевой с параметрами $n$ и $b$. А так как $n^{m} d^{n} \rightarrow 0$ при $n \rightarrow \infty$ при любом $m>0$, при $n \rightarrow \infty$ справедливо соотношение

$$
\mathbf{E}(x(n))=(n+1) b+c+O\left(d^{n}\right)=(n+1) b+c+o\left(n^{-m}\right) .
$$

Легко проверить также, что

$$
|\mathbf{E}(x(n))-((n+1) b+c)| \leqslant|d|^{n+1} /(1-d), \quad|\mathbf{E}(x(n))-n b| \leqslant 1 /(1-d) .
$$

Правые части этих неравенств могут быть большими при значениях $d$, близких к 1 .

\section{8. Дисперсия числа единиц}

8.1. Последовательно дифференцируя равенство (14) и разлагая вторую производную при $s=1$ в ряд по $v$, после некоторых преобразований получаем равенства

$$
\begin{aligned}
& f_{s}^{\prime}(1, v)=\frac{a+(1-a-q) v}{(1-d v)(1-v)^{2}}=\sum_{n=0}^{\infty}\left((n+1) b+c\left(1-d^{n+1}\right)\right) v^{n}=\sum_{n=0}^{\infty} f_{(n)}^{\prime}(1) v^{n}, \\
& f_{s s}^{\prime \prime}(1, v)=f_{s}^{\prime}(1, v) \frac{2(p-d v) v}{(1-v)(1-d v)}=2 f_{s}^{\prime}(1, v) \sum_{n=1}^{\infty}\left(b+(1-b) d^{n}\right) v^{n}=\sum_{n=1}^{\infty} f_{(n)}^{\prime \prime}(1) v^{n} .
\end{aligned}
$$

Вычисляя коэффициенты $f_{(n)}^{\prime}(1), f_{(n)}^{\prime \prime}(1)$ полученных рядов, находим, что

$$
\begin{aligned}
\mathbf{D}(x(n))= & f_{(n)}^{\prime \prime}(1)+f_{(n)}^{\prime}(1)-\left(f_{(n)}^{\prime}(1)\right)^{2}=2 \sum_{m=1}^{n}\left[\left((n-m+1) b+c\left(1-d^{n-m+1}\right)\right.\right. \\
& \left.\times\left(b+(1-b) d^{m}\right)\right]+(n+1) b+c\left(1-d^{n+1}\right)-\left((n+1) b+c\left(1-d^{n+1}\right)\right)^{2} .
\end{aligned}
$$


Суммируя и выделяя главную часть, получаем формулу для дисперсия

$$
\mathrm{D}(x(n))=a(1-a)+n b(1-b) \gamma+A+R(n),
$$

где

$$
\begin{aligned}
\gamma & =\frac{1+d}{1-d}, & A & =-\left(c^{2}(2-d)-c(1-2 b) \frac{3-d}{1-d}+2 b(1-b) \frac{d}{(1-d)^{2}}\right) d, \\
B & =-2 c(1-2 b) d, & C & =\left(2 c^{2}-c(1-b) \frac{3-d}{1-d}+2 b(1-b) \frac{d}{(1-d)^{2}}\right) d, \\
D & =-c^{2} d^{2}, & R(n) & =B n d^{n}+C d^{n}+D d^{2 n} .
\end{aligned}
$$

8.2. Заметим, что при $n \rightarrow \infty$

$$
R(n)=\left(B+\frac{C}{n}+\frac{D}{n} d^{n}\right) n d^{n}=O\left(n d^{n}\right) .
$$

По предположению $|d|<1$ и $n^{m} d^{n} \rightarrow 0$ при $n \rightarrow \infty$ при любом $m>0$, поэтому при $n \rightarrow \infty$

$$
\begin{aligned}
\mathrm{D}(x(n)) & =a(1-a)+n b(1-b) \gamma+A+O\left(n d^{n}\right) \\
& =a(1-a)+n b(1-b) \gamma+A+o\left(n^{-m}\right), \\
\mathrm{D}(x(n)) & \sim a(1-a)+n b(1-b) \gamma+A \sim n b(1-b) \gamma .
\end{aligned}
$$

Слагаемые $a(1-a)+n b(1-b) \gamma$ составляют биномиальную часть формулы (19) с коэффициентом искажения $\gamma$, учитывающим зависимость между случайными переменными $\xi(k)$. Слагаемые $A+B n d^{n}+C d^{n}+D d^{2 n}$ являются аддитивной поправкой на их зависимость и нестационарность.

8.3. Для стационарной последовательности ( $a=b, c=0)$ формулы (18), (19) дают следующие выражения:

$$
\begin{aligned}
& \mathbf{E}(x(n))=t(n+1) b, \\
& \mathbf{D}(x(n))=(n+1) b(1-b) \gamma-\frac{2 b(1-b) d}{(1-d)^{2}}\left(1-d^{n+1}\right) .
\end{aligned}
$$

Замечание 4. Главные члены $n b$ и $n b(1-b) \gamma$ формул для средних и дисперсий были выведены А. А. Марковым и использованы им в предельной теореме [1]. Другие члены формулы (19) могут играть существенную роль при значениях $d$, близких к 1 . Кроме того, они позволяют оценить точность нормального приближения и скорость сходимости к нему.

8.4. Оценим погрешность приближения $\mathrm{D}(x(n))$ ее членами $a(1-a)+n b(1-b) \gamma+A$ и $n b(1-b) \gamma$, используя равенство (19). Как нетрудно проверить, при $|d|<1$ верны неравенства

$$
|A| \leqslant \frac{5}{2(1-d)^{2}}, \quad|B| \leqslant \frac{2}{(1-d)^{2}}, \quad|C| \leqslant \frac{3}{2(1-d)^{2}}, \quad|D| \leqslant \frac{1}{(1-d)^{2}},
$$


откуда

$$
\begin{aligned}
|\mathrm{D}(x(n))-(a(1-a)+n b(1-b) \gamma+A)| & \leqslant \frac{3}{n} \frac{1}{(1-d)^{2}}, \\
|\mathrm{D}(x(n))-n b(1-b) \gamma| & \leqslant \frac{1}{4}+\left(\frac{5}{2}+\frac{3}{n}\right) \frac{1}{(1-d)^{2}} .
\end{aligned}
$$

Последние неравенства верны, когда

$$
n \geqslant 2+\frac{9}{4 \ln ^{2}|d|}
$$

или $n \geqslant 2$ при $d=0$. Неравенство $n \geqslant 2$ обеспечивает выполнение неравенства $2+3 /(2 n)+1 / n^{2} \leqslant 3$, а неравенство $n \geqslant 9 /\left(4 \ln ^{2}|d|\right)$ вместе с неравенством $\ln n \leqslant(3 / 4) \sqrt{n}$ влекут неравенство $\left|n d^{n}\right| \leqslant 1 / n$ при $0<|d|<1$ :

$$
n \geqslant \frac{9}{4 \ln ^{2}|d|} \Rightarrow-\ln |d| \geqslant \frac{3 \sqrt{n}}{2 n} \Rightarrow-\ln |d| \geqslant \frac{2 \ln n}{n} \Rightarrow \frac{1}{|d|^{n}} \geqslant n^{2} \Rightarrow n|d|^{n} \leqslant \frac{1}{n}
$$

Замечание 5. Если $d \rightarrow 1$ и рассматриваемые переменные $\xi(k)$ сильно зависимы, то $1 /(1-d)^{2} \rightarrow \infty$ и предлагаемые для дисперсии оценки теряют смысл. Но в этом случае также $\gamma \rightarrow \infty$. Если $d \rightarrow 0$ и рассматриваемые переменные $\xi(k)$ слабо зависимы, то $1 /(1-d)^{2} \rightarrow 1$ и предлагаемые для дисперсии оценки упрощаются. В этом случае $\gamma \rightarrow 1$. Если $d<0$, то знаменателями в оценках можно пренебречь.

Из полученных для абсолютных погрешностей оценок выводятся оценки для относительных погрешностей. Они имеют порядок $1 / n$.

При больших значениях $n$ указанные приближения можно эффективно использовать для вычислений. Оценки и примеры показывают, что

$$
\overline{\mathbf{E}}(x(n))=b+n b+c, \quad \overline{\mathbf{D}}(x(n))=a(1-a)+n b(1-b) \gamma+A
$$

хорошо приближают $\mathrm{E}(x(n))$ и $\mathrm{D}(x(n))$ даже при сравнительно небольших значениях $n$.

\section{9. Среднее число 1-серий}

9.1. Дифференцирование равенства (17) по $t$ и разложение в ряд по степеням $v$ дают равенство

$$
\begin{aligned}
g_{t}^{\prime}(1, v) & =\frac{(1-p v)(a+(1-a-q) v)}{(1-v)^{2}(1-d v)}=a+(1-(1-a) q) v \\
+ & \sum_{n=2}^{\infty}\left(a+(1-a)(1-q) n-\sum_{m=0}^{n-2}(1-q)\left(1-q-a(1-d)(m+1) d^{n-m-2}\right)\right) v^{n}
\end{aligned}
$$

Среднее число 1-серий выражается формулой

$$
\mathbf{E}(y(n))=a+n b(1-p)-(a-b) b\left(1-d^{n}\right) .
$$


9.2. По предположению $|d|<1$, поэтому при $n \rightarrow \infty$

$$
\mathbf{E}(y(n)) \sim a+n b(1-p)-(a-b) b .
$$

А так как $n^{m} d^{n} \rightarrow 0$ при $n \rightarrow \infty$ при любом $m>0$, при $n \rightarrow \infty$

$$
\mathbf{E}(y(n))=a+n b(1-p)-(a-b) b+O\left(d^{n}\right)=a+n b(1-p)-(a-b) b+o\left(n^{-m}\right) .
$$

Легко проверить также, что

$$
\begin{aligned}
|\mathbf{E}(y(n))-(a+n b(1-p)-(a-b) b)| & \leqslant \frac{|d|^{n}}{(1-d)^{2}}, \\
|\mathbf{E}(y(n))-n b(1-p)| & \leqslant 1+\frac{1-d^{n}}{(1-d)^{2}}
\end{aligned}
$$

а также

$$
\begin{aligned}
|\mathbf{E}(y(n))-(a+n b(1-p)-(a-b) b)| & \leqslant|d|^{n}, \\
|\mathbf{E}(y(n))-n b(1-p)| & \leqslant 2-d^{n} .
\end{aligned}
$$

Первой группой оценок целесообразно пользоваться, когда $d<0$, второй при $d>0$.

\section{0. Дисперсия числа 1-серий}

10.1. Дифференцирование равенства (17) дает равенство

$$
g_{t t}^{\prime \prime}(1, v)=2(1-p)(1-q) \frac{a+(1-a-a p-q) v-(1-a-q) p v^{2}}{(1-v)^{3}(1-d v)^{2}} v^{2}
$$

Разлагая правую часть равенства в ряд по степеням $v$, вычисляя коэффициенты $g_{(n)}^{\prime \prime}(1)$, используя для дисперсии числа 1-серий формулу

$$
\mathbf{D}(y(n))=g_{(n)}^{\prime \prime}(1)+\mathbf{E}(y(n))-\mathbf{E}(y(n))^{2},
$$

после некоторых преобразований приходим к равенству

$$
\mathbf{D}(y(n))=a(1-a)+n b(1-b) \bar{\gamma}+\bar{A}+\bar{R}(n)
$$

где

$$
\begin{aligned}
\bar{\gamma} & =p(b-q)+q(1-b) \\
\bar{A} & =b\left((a-b)^{2}(2-b)-(a-b)(1-2 b)(3-2 b)-2(1-b) b^{2}\right) \\
\bar{B} & =-2(a-b) b^{2}(1-q)(1+d) \\
\bar{C} & =b\left(-2(a-b)^{2}(1-b)+(a-b)(1-2 b)(3-2 b)+2(1-b) b^{2}\right) \\
\bar{D} & =-(a-b)^{2} b^{2} \\
\bar{R}(n) & =\bar{B} n d^{n-1}+\bar{C} d^{n}+\bar{D} d^{2 n}
\end{aligned}
$$


10.2. Заметим, что при $n>0$

$$
\bar{R}(n)=\left(\bar{B}+\frac{\bar{C} d}{n}+\frac{\bar{D} d^{n+1}}{n}\right) n d^{n-1}=O\left(n d^{n-1}\right) .
$$

Как нетрудно проверить,

$$
|\bar{A}| \leqslant 2 / 5, \quad|\bar{B}| \leqslant 27 / 128, \quad|\bar{C}| \leqslant 1, \quad|\bar{D}| \leqslant 1, \quad t\left|\bar{B}+\frac{\bar{C} d}{n}+\frac{\bar{D} d^{n+1}}{n}\right| \leqslant \frac{27}{128}+\frac{2}{n} .
$$

10.3. Для стационарной последовательности $(a=b)$ формулы (20), (21) дают равенства

$$
\begin{aligned}
& \mathbf{E}(y(n))=b+n b(1-p), \\
& \mathbf{D}(y(n))=(1-b) b\left(1+n \bar{\gamma}-2 b^{2}\left(1-d^{n}\right)\right) .
\end{aligned}
$$

В случае последовательности Бернулли, когда $a=b=p=1-q$ и $d=0$, эти равенства при $n \geqslant 1$ приобретают вид ([6])

$$
\begin{aligned}
& \mathbf{E}(y(n))=p(1+n(1-p)) \\
& \mathbf{D}(y(n))=p(1-p)\left(1-2 p^{2}+n\left(1-3 p+3 p^{2}\right)\right)
\end{aligned}
$$

Замечание 6. В [12] аналогичные выражения для средних и дисперсий указаны как следствия общих комбинаторных формул для конечных цепей Маркова из [10], [11]. Для вывода нужных выражений можно использовать также общие результаты, доказанные в книге [13].

\section{1. Ковариации}

11.1. Найдем $\mathrm{E}(x(n) y(n))$. Для этого продифференцируем производящую функцию $h(s, t, v)$ по $s$ и $t$, взяв значение производной в точке $s=1, t=1$ :

$$
h_{s t}^{\prime \prime}(1,1, v)=\frac{(a+(1-a-q) v)\left(1-(1+d) v+(2 p q-d) v^{2}\right)}{(1-v)^{3}(1-d v)^{2}} .
$$

Разлагая полученное выражение в ряд по степеням $v$, получаем равенства

$$
\begin{aligned}
& h_{s t}^{\prime \prime}(1,1, v)=\left(a+(1-q-a(p+q+1)) v+(q d-p+a(1+2 p q)) v^{2}\right. \\
& \left.\quad+(1-a-q)(2 p q-d) v^{3}\right) \sum_{k=0}^{\infty}(k+1) d^{k} v^{k} \sum_{n=0}^{\infty} \frac{(n+2)(n+1)}{2} v^{n}=\sum_{n=0}^{\infty} h_{(n)}^{\prime \prime}(1,1) v^{n}
\end{aligned}
$$

После некоторых преобразований находим, что

$$
h_{(n)}^{\prime \prime}(1,1)=\mathbf{E}(x(n) y(n))=n^{2} b^{2}(1-p)+\hat{A} n+\hat{B}+\hat{R}(n),
$$


где

$$
\begin{aligned}
\hat{A} & =b(2(a-b)(1-b)+1-b(1-b)(1+d)) \\
\hat{B} & =a+c(d-2 b(1-b)(1+d))+2 b^{2}(1-b) \frac{d}{1-d} \\
\hat{C} & =2(a-b) b(1-b) \\
\hat{D} & =-c(d-2 b(1-b)(1+d))-2 b^{2}(1-b) \frac{d}{1-d} \\
\hat{R}(n) & =\hat{C} n d^{n}+\hat{D} d^{n}
\end{aligned}
$$

11.2. Заметим, что при $n \geqslant 1$

$$
\hat{R}(n)=(\hat{C}+\hat{D} / n) n d^{n}=O\left(n d^{n}\right) .
$$

Как нетрудно проверить,

$$
|\hat{C}| \leqslant 8 / 27, \quad|\hat{D}| \leqslant 1 /(1-d), \quad|\hat{C}+\hat{D} / n| \leqslant 8 / 27+1 /((1-d) n) .
$$

11.3. Для стационарной последовательности $(a=b)$ коэффициенты в выражении (22) существенно упрощаются:

$$
\begin{aligned}
\hat{A} & =b(1-b(1-b)(1+d)), \\
\hat{B} & =b\left(1+2 b(1-b) \frac{d}{1-d}\right), \\
\hat{R}(n) & =-2 b^{2}(1-b) \frac{d^{n+1}}{1-d}
\end{aligned}
$$

В случае последовательности Бернулли, когда $a=b=p$ и $d=0$, эти равенства принимают вид

$$
\hat{A}=p(1-p(1-p)), \quad \hat{B}=p, \quad \hat{R}(n)=0 .
$$

11.4. Вычислим ковариацию $\operatorname{Cov}(x(n), y(n))$ числа 1-событий и 1-серий. Подставляя В формулу

$$
\operatorname{Cov}(x(n), y(n))=\mathbf{E}(x(n) y(n))-\mathbf{E}(x(n)) \mathbf{E}(y(n))
$$

найденные значения $\mathrm{E}(x(n) y(n)), \mathrm{E}(x(n)), \mathrm{E}(y(n))$, после некоторых преобразований получаем равенство

$$
\operatorname{Cov}(x(n), y(n))=n b(1-b)(1-2 b)+C_{0}+R C(n),
$$

где

$$
\begin{aligned}
C_{0} & =(1-b)\left(c(1-a-2 b-b d)+b+2 b^{2} d /(1-d)\right), \\
C_{1} & =c b\left(2 q-p q-p^{2}\right), \\
C_{2} & =-c(a(b-(1-b) d)-2 b(1-b)+(1-2 b) d)-2 b^{2}(1-b) d /(1-d), \\
C_{3} & =(a-b) c b d, \\
R C(n) & =C_{1} n d^{n}+C_{2} d^{n}+C_{3} d^{2 n} .
\end{aligned}
$$


11.5. Заметим, что

$$
R C(n)=\left(C_{1}+\frac{C_{2}}{n}+\frac{C_{3}}{n} d^{n}\right) n d^{n}=O\left(n d^{n}\right)
$$

Как нетрудно проверить,

$$
\left|C_{1}\right| \leqslant 1, \quad\left|C_{2}\right| \leqslant \frac{3}{1-d}, \quad\left|C_{3}\right| \leqslant \frac{1}{1-d}, \quad\left|C_{1}+\frac{C_{2}}{n}+\frac{C_{3}}{n} d^{n}\right| \leqslant 1+\frac{4}{n(1-d)} .
$$

11.6. Для стационарной последовательности $(a=b)$ коэффициенты в выражении для ковариации существенно упрощаются:

$$
C_{0}=b(1-b) \frac{1-(1-2 b) d}{1-d}, \quad R C(n)=-2 b^{2}(1-b) \frac{d^{n+1}}{1-d}
$$

В случае последовательности Бернулли, когда $a=b=p$ и $d=0$, эти равенства принимают вид

$$
C_{0}=p(1-p), \quad R C(n)=0 .
$$

В симметричном случае, когда $p=q$ и $b=1 / 2$,

$$
\operatorname{Cov}(x(n), y(n))=C_{0}+C_{1} n d^{n}+C_{2} d^{n}+C_{3} d^{2 n},
$$

где

$$
\begin{aligned}
& C_{0}=\frac{2-(1-2 a)(1-2 a-2 p)}{16(1-p)}, \\
& C_{1}=\frac{(2 a-1) p}{4} \\
& C_{2}=\frac{(1-a)(2 a(1-p)-p)}{4(1-p)}, \\
& C_{3}=-\frac{(1-2 a)^{2}(1-2 p)}{16(1-p)} .
\end{aligned}
$$

Замечание 7. Таким образом, в несимметричном и симметричном случаях соответственно

$$
\operatorname{Cov}(x(n), y(n))=O(n), \quad \operatorname{Cov}(x(n), y(n))=O(1)
$$

11.7. Вычислим коэффициент корреляции $K(x(n), y(n))$ случайных величин $x(n), y(n)$. Подставляя в формулу

$$
K(x(n), y(n))=\frac{\operatorname{Cov}(x(n), y(n))}{\sqrt{\mathrm{D}(x(n)) \mathrm{D}(y(n))}}
$$

найденные значения $\operatorname{Cov}(x(n), y(n)), \mathbf{D}(x(n)), \mathbf{D}(y(n))$, после некоторых преобразований получаем, что

$$
K(x(n), y(n))=\frac{1}{b(1-b) \sqrt{(p+q)\left(p b^{2}+q(1-b)^{2}\right)}}(b(1-b)(1-2 b)+R K(n)),
$$


и $|R K(n)| \leqslant \bar{r} / n$ при

$$
n \geqslant 6 \sqrt{3}\left(\frac{(\bar{r})^{3 / 4}}{\sqrt[3]{(\bar{r})^{33 / 20}-1 / 30}}\right)^{5}
$$

где

$$
\begin{aligned}
& \bar{r}=\left(\frac{r_{0}}{2}+\frac{1}{6 \sqrt{3}}\right)\left(5 / 2+r_{1}+r_{2}+r_{1} r_{2}\right) \\
& r_{0}=\left|C_{0}+R C(n)\right| \leqslant \frac{1}{3(1-d)}+n|d|^{n}+\frac{3|d|^{n}}{1-d}+\frac{d^{2 n}}{1-d} \\
& r_{1}=\left|\frac{a(1-a)+A+R(n)}{b(1-b) \gamma}\right| \leqslant \frac{6}{b(1-b)\left(1-d^{2}\right)}+\frac{2 n|d|^{n+1}+4|d|^{n+1}+d^{2 n+2}}{b(1-b)\left(1-d^{2}\right)} \\
& r_{2}=\left|\frac{a(1-a)+\bar{A}+\bar{R}(n)}{b(1-b) \bar{\gamma}}\right| \leqslant \frac{3}{5 b(1-b) \bar{\gamma}}+\frac{(27 / 128) n|d|^{n-1}+|d|^{n}+d^{2 n}}{b(1-b) \bar{\gamma}}
\end{aligned}
$$

Замечание 8. Таким образом, в симметричном случае

$$
K(x(n), y(n))=O(1 / n)
$$

В длинных двоичных марковских последовательностях с симметричным распределением число единиц и число 1-серий асимптотически некоррелированы. Это соответствует представлению о рассматриваемой марковской последовательности $\xi(1), \ldots, \xi(n)$ как об асимптотически бернуллиевой.

\section{2. Нормальное приближение}

12.1. Равенство (5) для совместного распределения случайных переменных $x(n), y(n)$ позволяет получить достаточно простую формулу для его нормального приближения. Будем использовать приближенные формулы для биномиального распределения, указанные в теореме 1.1 .12 в [14] и [15]. Введем обозначения

$$
\begin{aligned}
\sigma(n, p) & =\sqrt{n p(1-p)}, & x & =x(k, n, p)=(k-n p) / \sigma(n, p), \\
\Delta(x, n, p) & =\frac{(1-2 p)\left(3 x-x^{3}\right)}{6 \sigma(n, p)}, & \varepsilon(x, n, p) & =C \frac{1+x^{6}}{\sigma^{2}(n, p)} \frac{1}{\sqrt{2 \pi} \sigma(n, p)} e^{-x^{2} / 2}, \\
u & =x(j-1, i-1,1-p), & v & =v(\alpha, \beta)=x(j-\alpha-\beta, n-i, 1-q) .
\end{aligned}
$$

В этих обозначениях

$$
b(k, n, p)=\frac{1}{\sqrt{2 \pi} \sigma(n, p)} e^{-x^{2} / 2}(1+\Delta(x, n, p))+\varepsilon(x, n, p),
$$

когда $n \rightarrow \infty,\left(1+x^{6}\right) / \sigma^{2}(n, p) \rightarrow 0$.

Для всех $x$ из данного отрезка вещественной прямой при $\sigma(n, p) \geqslant 25$ верно неравенство

$$
|\varepsilon(x, n, p)| \leqslant \frac{0,15+0,25|1-2 p|}{\sigma^{3}(n, p)}+e^{-3 \sigma(n, p) / 2} .
$$


Используя равенства (23) и (5) получаем, что при $n \rightarrow \infty,\left(1+x^{6}\right) / \sigma^{2}(n, p) \rightarrow 0$

$$
\begin{aligned}
P_{\alpha \beta}(i, j, n)= & \chi_{\alpha \beta} b(j-1, i-1,1-p) b(j-\alpha-\beta, n-i, 1-q) \\
= & \frac{\chi_{\alpha \beta}}{2 \pi \sigma(i-1, p) \sigma(n-i, q)} e^{-\left(u^{2}+v^{2}\right) / 2}(1+\Delta(u, v, n, i, p, q)) \\
& \quad+\varepsilon(u, v, n, i, p, q),
\end{aligned}
$$

где

$$
\begin{aligned}
\Delta(u, v, n, i, p, q)= & \Delta(u, i-1, p)+\Delta(v, n-i, q)+\Delta(u, i-1, p) \Delta(v, n-i, q), \\
\varepsilon(u, v, n, i, p, q)= & \frac{1}{\sqrt{2 \pi} \sigma(n-i, q)} e^{-v^{2} / 2}(1+\Delta(v, n-i, q)) \varepsilon(u, i-1, p) \\
& +\frac{1}{\sqrt{2 \pi} \sigma(i-1, p)} e^{-u^{2} / 2}(1+\Delta(u, i-1, p)) \\
& \times \varepsilon(v, n-i, q)+\varepsilon(u, i-1, p) \varepsilon(v, n-i, q) .
\end{aligned}
$$

Для всех $x$ из данного отрезка вещественной прямой верно неравенство

$$
\begin{aligned}
|\varepsilon(u, v, n, i, p, q)| \leqslant & \frac{1}{\sqrt{2 \pi} \sigma^{3}(i-1, p) \sigma^{3}(n-i, q)}(B(q, n-i) A(p)+B(p, i-1) A(q) \\
& +\sqrt{2 \pi} A(p) A(q))+(B(q, n-i)+\sqrt{2 \pi} A(q)) \frac{e^{-3 \sigma(i-1, p) / 2}}{\sqrt{2 \pi} \sigma^{3}(n-i, q)} \\
& +(B(p, i-1)+\sqrt{2 \pi} A(p)) \frac{e^{-3 \sigma(n-i, q) / 2}}{\sqrt{2 \pi} \sigma^{3}(i-1, p)} \\
& +e^{-3(\sigma(i-1, p)+\sigma(n-i, q)) / 2},
\end{aligned}
$$

где

$$
A(x)=0,15+0,25|1-2 x|, \quad B(x, y)=2 x^{2}(1-x)^{2}+\frac{1}{3}|1-2 x| y .
$$

Более простая и грубая оценка имеет вид

$$
\begin{aligned}
|\varepsilon(u, v, n, i, p, q)| \leqslant & \frac{2(n+3)}{15 \sqrt{2 \pi} \sigma^{3}(i-1, p) \sigma^{3}(n-i, q)} \\
& +\left(\frac{5}{4}+\frac{1}{3}(n-i)\right) \frac{e^{-3 \sigma(i-1, p) / 2}}{\sqrt{2 \pi} \sigma^{3}(n-i, q)} \\
& +\left(\frac{5}{6}+\frac{i}{3}\right) \frac{e^{-3 \sigma(n-i, q) / 2}}{\sqrt{2 \pi} \sigma^{3}(i-1, p)}+e^{-3(\sigma(i-1, p)+\sigma(n-i, q)) / 2} .
\end{aligned}
$$

Оставляя только главные члены и разлагая на нормальные множители, находим, что

$$
\begin{aligned}
P_{\alpha \beta}(i, j, n) & \sim \frac{(\alpha a+(1-\alpha)(1-a))}{\sqrt{2 \pi} \sigma(i-1, p)} \exp \left(-\frac{(j-1-(i-1)(1-p))^{2}}{2 \sigma^{2}(i-1, p)}\right) \\
& \times \frac{(\beta(1-q)+(1-\beta)(1-p))}{\sqrt{2 \pi} \sigma(n-i, q)} \exp \left(-\frac{(j-\alpha-\beta-(n-i)(1-q))^{2}}{2 \sigma^{2}(n-i, q)}\right)
\end{aligned}
$$

при $i \rightarrow \infty,\left(1+u^{6}\right) / \sigma^{2}(i-1, p) \rightarrow 0, n-i \rightarrow \infty,\left(1+v^{6}\right) / \sigma(n-i, q) \rightarrow 0$. Асимттотическая формула (24) сразу следует из формулы (5) и классической теоремы Муавра-Лапласа. 
12.2. Нормальные приближения для распределения числа единиц в марковских последовательностях описаны во многих работах. Интегральная предельная теорема для двоичной последовательности была доказана А. А. Марковым в [1]. Интегральная и локальная предельные теоремы для марковской последовательности с конечным множеством значений были доказаны А. Н. Колмогоровым в [16].

Предельные теоремы для распределения числа 1-серий в двоичных марковских последовательностях можно вывести из локальной теоремы для чисел переходов в марковской последовательности с конечным множеством значений, доказанной в [10].

В двоичном случае формулировки и доказательства теорем существенно упрощаются.

\section{3. Случайные блуждания}

13.1. Рассмотрим последовательность случайных векторов $(x(n), y(n), \xi(n))$. Она является марковской со счетным множеством значений $C=\mathbf{Z}_{+} \times \mathbf{Z}_{+} \times\{0,1\}$, начальным распределением $\bar{P}=\left(\bar{p}_{\sigma}\right)$ и переходной матрицей $\bar{Q}=\left(\bar{q}_{\gamma}\right), \gamma, \Delta \in C$. Из определений следует, что

$$
\begin{aligned}
\bar{p}_{(0,0,0)} & =1-a, & \bar{p}_{(1,1,1)} & =a, \\
\bar{q}_{(i, j, 0),(i, j, 0)} & =q, & \bar{q}_{(i, j, 0),(i+1, j+1,1)} & =1-q, \\
\bar{q}_{(i, j, 1),(i+1, j, 1)} & =p, & \bar{q}_{(i, j, 1),(i, j, 0)} & =1-p,
\end{aligned}
$$

все остальные начальные и переходные вероятности равны 0.

13.2. Последовательность векторов $(x(n), y(n), \xi(n))$ описывает случайное блуждание по целочисленной пространственной решетке в бесконечном прямоугольнике $C=\mathbf{Z}_{+} \times \mathbf{Z}_{+} \times\{0,1\}$. Находясь в момент $n>0$ в точке $(i, j, 0)$, частица в момент $n+1$ может с вероятностью $q$ остаться в этой точке, а с вероятностью $1-q$ подняться по диагонали в точку $(i+1, j+1,1)$. Находясь в момент $n>0$ в точке $(i, j, 1)$, частица в момент $n+1$ может с вероятностью $p$ переместиться в точку $(i+1, j, 1)$, а с вероятностью $1-p$ упасть в точку $(i, j, 0)$. Таким образом, частица может оставаться на месте или перемещаться из данной вершины каждого единичного куба в другую по указанным правилам. Выйдя из точки, частица в нее уже не возвращается.

\section{4. Операторные уравнения}

14.1. Рассматриваемое случайное блуждание определяется системой рекуррентных уравнений и эквивалентным ей матричным уравнением специального вида. Составим операторное уравнение для распределения

$$
\bar{p}(n)=(p(i, j, k, n)), \quad(i, j, k) \in C, \quad p(i, j, k, n)=\mathbf{P}\{x(n)=i, y(n)=j, \xi(n)=k\}
$$

случайного вектора $(x(n), y(n), \xi(n))$. Из определений следует, что

$$
p(0,0,0,0)=1-a, \quad p(1,1,1,0)=a, \quad \bar{p}(n+1)=\bar{p}(n) \bar{Q}, \quad n \geqslant 0 .
$$

Рассмотрим банахово пространство $L^{1}=L^{1}(C)$ суммируемых числовых функций на множестве $C$. Ясно, что $\bar{p}(n) \in L^{1}$ и норма $|\bar{p}(n)|=1$. Матрица $Q$ представляет ограниченный линейный оператор в $L^{1}(C)$ и имеет норму 1. Такие операторы и линейные операторные уравнения подробно рассматривались в [18]. 
14.2. Пусть

$$
\hat{p}(u)=\sum_{n=0}^{\infty} \bar{p}(n) u^{n}, \quad|u|<1 .
$$

Так как $|\bar{p}(n)|=1$, векторный ряд $\bar{p}(n) u^{n}$ абсолютно суммируем при $|u|<1$ и функщия $\hat{p}$ определена внутри единичного круга. Умножая равенство для $\bar{p}(n)$ на $u^{n}$ и суммируя, получаем операторное уравнение для $\hat{p}$ :

$$
\begin{aligned}
\hat{p}(u)-\bar{p}(0) & =\hat{p}(u) \bar{Q} u, \\
\hat{p}(u)(I-\bar{Q} u) & =\bar{p}(0) .
\end{aligned}
$$

Здесь $I$ обозначает единичную $|C| \times|C|$ матрицу, которая представляет тождественное преобразование множества $C$. Так как $|\bar{Q} u|=|\bar{Q}||u|<1$, полученное уравнение корректно и его решение представляется рядом Неймана

$$
\hat{p}(u)=\bar{p}(0)(I-\bar{Q} u)^{-1}=\sum_{n=0}^{\infty} \bar{p}(0) \bar{Q}^{n} u^{n}
$$

Это соответствует равенству

$$
\bar{p}(n)=\bar{p}(0) \bar{Q}^{n}
$$

вытекающему из марковского свойства.

Дело сводится к исследованию степеней блочной матрицы $\bar{Q}$ или обратной матрищы $(I-\bar{Q} u)^{-1}$.

\section{Список литературы}

1. Марков А. А., Исчисление вероятностей. Императорская Академия Наук, Санкт-Петербург, 1913.

2. Колмогоров А. Н., Теория вероятностей и математическая статистика. Наука, Москва, 1986.

3. Сарымсаков Т. А., Основы теории прочессов Маркова. Гостехиздат, Москва, 1954.

4. Сираждинов С. Х., Предельные теоремы для иепей Маркова. Изд-во АН УзССР, Ташкент, 1955.

5. Савельев Л. Я., Случайные соответствия, двоичные матрицы и серии. Дискретная математика (1999) 11, №4, 3-26.

6. Mood A. M., The distribution theory of runs. Ann. Math. Stat. (1940) 11, №4, 367-392.

7. Hirano K., Aki S., On the number of occurrences of success runs of length $k$ in a two-state Markov chain. Statist. Sinica (1992) 3, 313-320.

8. Uchida M., On number of occurrences of success runs of specified length in a higher-order two-state Markov chain. Ann. Inst. Statist. Math. (1998) 50, №3, 587-601.

9. Феллер В., Введение в теорию вероятностей и ее приложения, т. 1. Мир, Москва, 1984.

10. Смирнов Н. В., Сарманов О. В., Захаров В. К., Локальная предельная теорема для чисел переходов в цепи Маркова и ее применения. Докл. АН СССР (1966) 167, №6, 1238-1241.

11. Сарманов О. В., Захаров В. К., Комбинаторная задача Н. В. Смирнова. Докл. АН СССР (1967) 176, №3, 530-532.

12. Захаров В. К., Сарманов О. В., О законе распределения числа серий в однородной цепи Маркова. Докл. АН СССР (1968) 179, №3, 526-528. 
13. Романовский В. И., Дискретные чепи Маркова. Гостехиздат, Москва, 1949.

14. Колчин В. Ф., Случайные графы. Физматлит, Москва, 2000.

15. Гамкрелидзе Н. Г., Муавра-Лапласа теорема. В кн.: Вероятность и математическая статистика. Энииклопедия. Большая Российская энциклопедия, Москва, 1999.

16. Колмогоров А. Н., Локальная предельная теорема для классических цепей Маркова. Известия AН СCCP. Сер. матем. (1949) 13, 281-300.

17. Савельев Л. Я., Серии в марковских последовательностях. Сиб. матем. ж. (1991) 32, №4, 116-132.

18. Савельев Л. Я., Марковские матричные операторы. Деп. ВИНИТИ, №457-В 95, 1995.

Статья поступила 12.10.2003. 